\title{
On-site cycling drag analysis with the Ring of Fire
}

\author{
Alexander Spoelstra $^{1}\left[{ }^{10} \cdot\right.$ Luigi de Martino Norante $^{1} \cdot$ Wouter Terra $^{1} \cdot$ Andrea Sciacchitano $^{1} \cdot$ Fulvio Scarano $^{1}$
}

Received: 8 November 2018 / Revised: 12 April 2019 / Accepted: 23 April 2019 / Published online: 13 May 2019

(c) The Author(s) 2019

\begin{abstract}
The Ring of Fire (RoF) measurement concept, introduced by Terra et al. (Exp Fluids 58:83. https://doi.org/10.1007/s0034 8-017-2331-0, 2017; Experiments in Fluids 59:120, 2018), is applied to real cyclists to enable the aerodynamic drag determination during sport action. This principle is based on large-scale stereoscopic particle image velocimetry (PIV) measurements over a plane crossed by the athlete during cycling. The momentum before and after the passage of the athlete poses the basis for the control volume analysis in the athlete's frame of reference, which returns the aerodynamic drag. This approach extrapolates aerodynamic studies towards more realistic conditions, compared to experiments performed in wind tunnels with scaled or stationary athletes. The measurement concept is termed Ring of Fire as the rider crosses a region of intense light. Two experiments are conducted, indoor and outdoor, with attention placed on the effects of the environmental conditions and the confinement of the measurement region. Stereo-PIV measurements feature a plane of approximately $2 \times 2 \mathrm{~m}^{2}$, using neutrally buoyant sub-millimeter helium-filled soap bubbles (HFSB) as flow tracers. The drag measurement is obtained examining the wake produced by the athlete. It is observed that the drag value becomes independent of time after about 5 torso lengths from the passage. A statistical estimate of the drag is produced combining the results of several passages. Fluctuations of the drag value during a single passage are associated with the unsteady wake flow. Overall fluctuations among different transits are ascribed to the varying conditions of the airflow prior to the passage of the athlete. The experiments conducted outdoor exhibit significantly larger dispersion of the drag value, compared to the quieter conditions indoor. Repetition of the transit 10-30 times yields a basis for statistical convergence of the average drag value. The flow topology past the cyclist compares satisfactorily between both experiments and with wind tunnel experiments reported in literature. The current measurements clearly separate drag values from upright and time-trial athlete's positions, indicating the suitability of this principle for aerodynamic analysis and optimization studies.
\end{abstract}

\section{Graphical abstract}

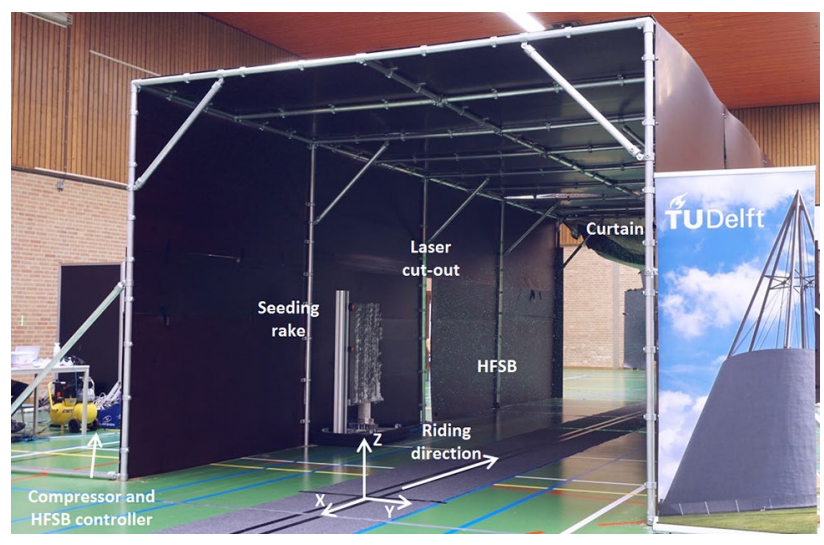

Electronic supplementary material The online version of this article (https://doi.org/10.1007/s00348-019-2737-y) contains supplementary material, which is available to authorized users.

Extended author information available on the last page of the article 


\section{Introduction}

Most experimental research in sport aerodynamics is performed in wind tunnels, despite the fact that the dynamical situation to be simulated (e.g., a cycling or running athlete) poses challenges related to the athlete motion and its control. As a result, the problem is often simplified reverting to a stationary scaled model to match the constraints posed by the wind tunnel size and the measurement techniques used for the aerodynamic analysis. The aerodynamic force is directly measured by connecting the model to a force balance. Alternatively, the drag force can be derived from velocity measurements in the wake of the object, carried out either via Pitot rakes (e.g., Jones 1936) or particle image velocimetry more recently (Kurtulus et al. 2007; van Oudheusden et al. 2007; David et al. 2009). The latter principle invokes conservation of momentum in a control volume that encloses the object. The deficit of momentum flux past the object corresponds to the aerodynamic drag acting on it (van Oudheusden et al. 2006).

In some cases, wind tunnel experiments cannot reproduce the aerodynamic conditions with sufficient accuracy and experiments whereby the model moves in quiescent air are considered. Typical limitations encountered in wind tunnel tests of sport aerodynamics range from inaccurate scaling of shape and roughness, model blockage, interference of the support (Barlow et al. 1999) or, for instance, when the flow behind accelerating objects is to be dealt with (Coutanceau and Bouard 1977). Additionally, the wake development far downstream of the model can be performed with more advantages when the model is towed (Scarano et al. 2002) or catapulted in quiescent fluid (Von Carmer et al. 2008). The transiting model approach has been successfully adopted to investigate also ground vehicle aerodynamics (Jönsson and Loose 2016) or animals in free flight (Hedenström and Johansson 2015; Ben-Gida et al. 2013).

The recent works of Terra et al. $(2017,2018)$ with tomographic PIV in combination with helium-filled soap bubbles to determine the drag of a towed sphere can be seen as preliminary to the current study. The use of HFSB offers the potential to upscale the measurement region up to several square meters as demonstrated by Bosbach et al. (2009).

In the present study, a measurement apparatus is realized that quantifies the aerodynamic drag of a full-scale cyclist during sport action. The experimental procedure to achieve drag measurements follows the same principles discussed by Terra et al. (2017). The measurements are performed by large-scale stereoscopic PIV over a field of view of about $4 \mathrm{~m}^{2}$. For such approach, where the rider crosses the illuminated measurement plane, the experimental method is referred with the name "Ring of Fire" (RoF).
Here, the RoF concept is applied for the study of the aerodynamic drag in cycling. The latter dominates the forces opposing the athlete's motion, which justifies the attention devoted to aerodynamic drag in several studies (Kyle and Burke 1984; Wilson 2004; Lukes et al. 2005; Crouch et al. 2017 among others). The flow field around a pedaling cyclist features a complex system of vortices, in turn depending also upon the cyclist's torso and legs position along the crank cycle. These latter variations in aerodynamic drag cannot be solely ascribed to changes in frontal area, but result from complex aerodynamic interactions leading to different flow and vortex topology. Crouch et al. (2014) have produced a detailed aerodynamic survey in the wake of a cyclist by wind tunnel experiments. The work resulted in the identification of the most prominent streamwise vortices emanating from the athlete at different positions during pedaling.

The detailed velocity field around a full-scale cyclist model has been recently measured with robotic particle image velocimetry (Jux et al. 2018), where also the near field flow topology has been characterized.

The RoF experiments aim at determining the aerodynamic drag of the cyclist during sport action, such to obtain an estimate close to on-site conditions. The results are to be compared not only to the above mentioned wind tunnel studies and computational fluid dynamics (CFD) simulations, but also to other techniques currently practiced for on-site measurements (coast down, Petrushov 1998; torque power output, Grappe et al. 1997). Moreover, they support the correlation between the aerodynamic drag and the flow field by quantitative visualizations of the velocity field in the cyclist's wake.

The present work describes the realization of the RoF concept for full-scale sport aerodynamics and discusses the experimental procedures for indoor and outdoor experiments, mimicking, respectively, track and road cycling. The aerodynamic drag estimation from cyclists during sport action is compared to literature data from wind tunnel experiments and other techniques.

Furthermore, the experiments cover different postures of the cyclist (time trial and upright) with the aim to directly measure the effect of posture on aerodynamic drag and its detectability with the RoF.

\section{Working principle}

The aerodynamic drag of an object moving in a fluid can be evaluated invoking the conservation of momentum expressed in a control volume. A recent review of the problem has been provided by Rival and Van Oudheusden (2017). The formulation of the problem is simplified from its unsteady form to the steady condition when applying a Galilean transformation (Arnold 1989), whereby the frame 
of reference moves with the object and the fluid is considered in uniform motion upstream of the object (Terra et al. 2018). If the control surfaces $S_{1}$ and $S_{2}$ are sufficiently far from the object surface, it can be shown that the viscous stress is negligible (Kurtulus et al. 2007). The drag force can, thus, be expressed as follows:

$$
\begin{aligned}
D(t) & =\underbrace{\rho \iint_{S_{1}} U_{\infty}^{2} \mathrm{~d} S+\iint_{S_{1}}\left(p_{\infty}-p_{1}\right) \mathrm{d} S}_{1} \\
& -\rho \iint_{2} \underbrace{}_{S_{2}} U_{\infty}^{2} \mathrm{~d} S+\iint_{S_{2}}\left(p_{\infty}-p_{2}\right) \mathrm{d} S
\end{aligned} .
$$

In the expression above $\rho$ is the air density. The cyclist is assumed to move at constant speed $u_{\mathrm{C}}$ with respect to the laboratory frame of reference. Let us now consider a control surface normal to the direction of motion of the cyclist. Prior to the passage of the cyclist, the air motions feature a chaotic velocity $u_{\text {env }}$, resulting from the environmental effects, as depicted in Fig. 1-top. Assuming uniform and quiescent conditions prior to the passage would largely simplify the problem formulation. However, even in scaled experiments, the disturbances in the air motion induced by the environment and the seeding generation are reported not to be negligible (Terra et al. 2018).
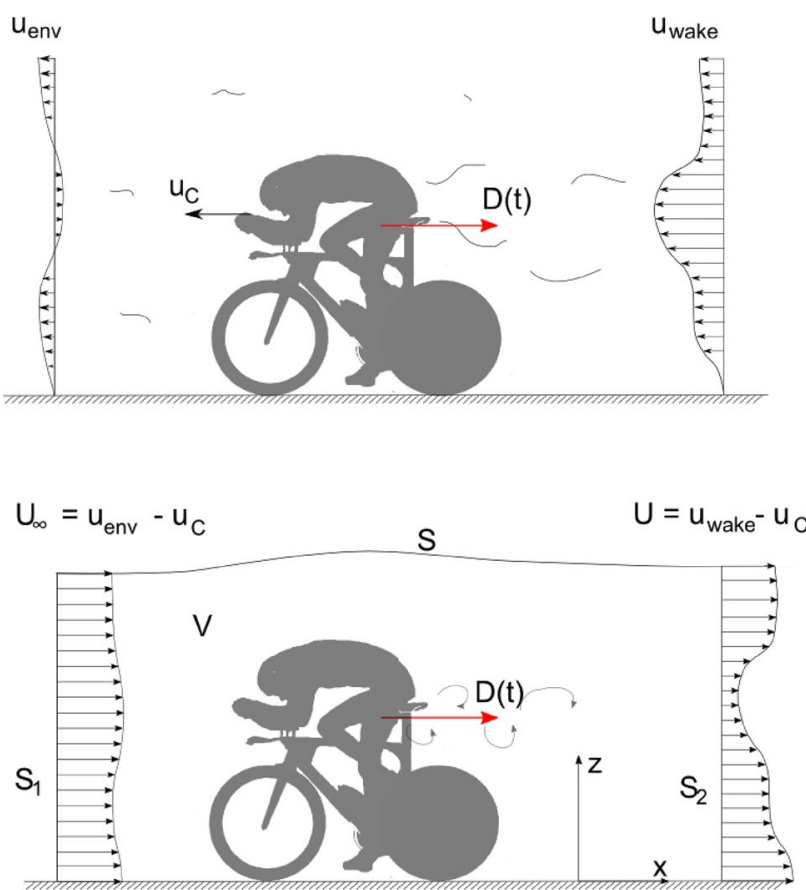

Fig. 1 Schematic view of the velocity distribution before and after the passage of the cyclist (top). Same view after Galilean transformation in the cyclist frame of reference (bottom)
After the passage of the cyclist, the flow velocity features a coherent wake with a velocity profile $u_{\text {wake }}$ that follows the moving cyclist. Making use of a Galilean transformation, the representation of velocity and momentum changes from the laboratory to the cyclist frame of reference moving at speed $u_{C}$. As a result, the air flow velocity ahead of the cyclist can be written as $U_{\infty}=u_{\text {env }}-u_{\mathrm{C}}$, while that at its back as (Fig. 1-bottom):

$$
\begin{aligned}
& D(t)=\underbrace{\rho \iint_{S_{1}}\left(u_{\mathrm{env}}-u_{\mathrm{C}}\right)^{2} \mathrm{~d} S+\iint_{S_{1}}\left(p_{\infty}-p_{1}\right) \mathrm{d} S}_{1} \\
& -\underbrace{-\rho \iint_{S_{2}}\left(u_{\text {wake }}-u_{\mathrm{C}}\right)^{2} \mathrm{~d} S-\iint_{S_{2}}\left(p_{\infty}-p_{2}\right) \mathrm{d} S}_{2} .
\end{aligned}
$$

This expression is valid at the condition that the mass flow is conserved across $S_{1}$ and $S_{2}$. This is ensured by shrinking the inlet plane $\left(S_{1}\right)$ from the outer edges, starting from the equal size as that of the outlet plane $\left(S_{2}\right)$.

Equation (2) yields the instantaneous aerodynamic drag from the surface integral of momentum and pressure over a fixed plane before and after passage of the cyclist. Ensemble averaging (Eq. 3) of the drag among multiple passages is performed to achieve a higher degree of statistical convergence.

$\bar{D}(t)=\frac{1}{N} \sum_{j=1}^{N} D_{j}(t)$,

where $N$ is the number of model passages. The aerodynamic drag exhibits temporal fluctuations associated with the unsteady nature of the flow around the cyclist. However, these unsteady fluctuations are little relevant to the evaluation of the cyclist's drag, given their short time scale. Time averaging is, therefore, performed within the ensemble average (Eq. 4) with the objective of reducing the effect of the unsteady fluctuations on the evaluation of the time-average drag.

$\overline{\bar{D}}=\frac{1}{T} \sum_{i=1}^{T} \bar{D}\left(t_{i}\right)$

where $T$ is the total number of time steps and $\bar{D}\left(t_{i}\right)$ is the ensemble average drag at each time step in the wake.

\section{Experimental setup and procedure}

Experiments were conducted with a cyclist riding a timetrial (TT) bike. For the indoor case, the cyclist was male, $1.89 \mathrm{~m}$ tall, with weight of $68 \mathrm{~kg}$. He wore a short-sleeve 
time-trial suit from Team Sunweb and a Giant Rivet TT helmet. The cyclist riding through the outdoor setup was $1.84 \mathrm{~m}$ tall and weighed $83 \mathrm{~kg}$ at the moment of testing. He was equipped with a long sleeved time-trial suit from Team Blanco and a Lazer Wasp TT helmet. Moreover, for safety reasons, both cyclists wore a pair of laser goggles. The approximate torso chord length, c, for both athletes, is $600 \mathrm{~mm}$. In the indoor experiment, a Giant Trinity TT Advanced Pro bike with $2 \times 11$ gears was used, while a Ridley Cheetah TT bike with $2 \times 9$ gears was used for the outdoor experiment.

\subsection{Experimental facilities and cycling conditions}

The experimental facilities and test conditions are presented in Table 1. The top view of the sport hall and of the outdoor site is shown in Fig. 2. The flow tracers are generated and confined within a tunnel of $4 \mathrm{~m} \times 3 \mathrm{~m}$ and $3 \mathrm{~m} \times 2 \mathrm{~m}$ (width $\times$ height) for the indoor and outdoor experiments, respectively. Curtains are used to maintain a high concentration of tracers within the duct. The entrance and the exit in the outdoor experiment are closed during accumulation and opened prior to the transit of the cyclist. For the indoor experiment, a curtain at the exit was sufficient. The measurement plane is near the half of the duct. Considering the small blockage ratios of 3.5 and $7 \%$ for the indoor and outdoor experiments, respectively, a non-confined environment
Table 1 Overview of experimental parameters and conditions
Fig. 2 Top-view schematics of the indoor (top) and outdoor (bottom) field facility

\begin{tabular}{lll}
\hline & Indoor & Outdoor \\
\hline Duct dimensions $\left(\mathrm{m}^{3}\right)$ & $10 \times 4 \times 3[x, y, z]$ & $6 \times 3 \times 2[x, y, z]$ \\
Solid blockage $(\%)$ & 3.5 & 7 \\
Cyclist velocity $\left(u_{\mathrm{C}}\right)(\mathrm{m} / \mathrm{s})$ & $5.3 \pm 0.1$ & $8.3 \pm 0.2$ \\
Reduced pedaling frequency $(k)$ & 0.12 & 0.23 \\
Crank length $(\mathrm{mm})$ & 175 & 172.5 \\
Zero degree crank & Horizontal, right front & Horizontal, right front \\
Acceleration length $(\mathrm{m})$ & 25 & 30 \\
Deceleration length $(\mathrm{m})$ & 10 & 40 \\
Torso based Reynolds number & $2.1 \times 10^{5}$ & $3.2 \times 10^{5}$ \\
\hline
\end{tabular}

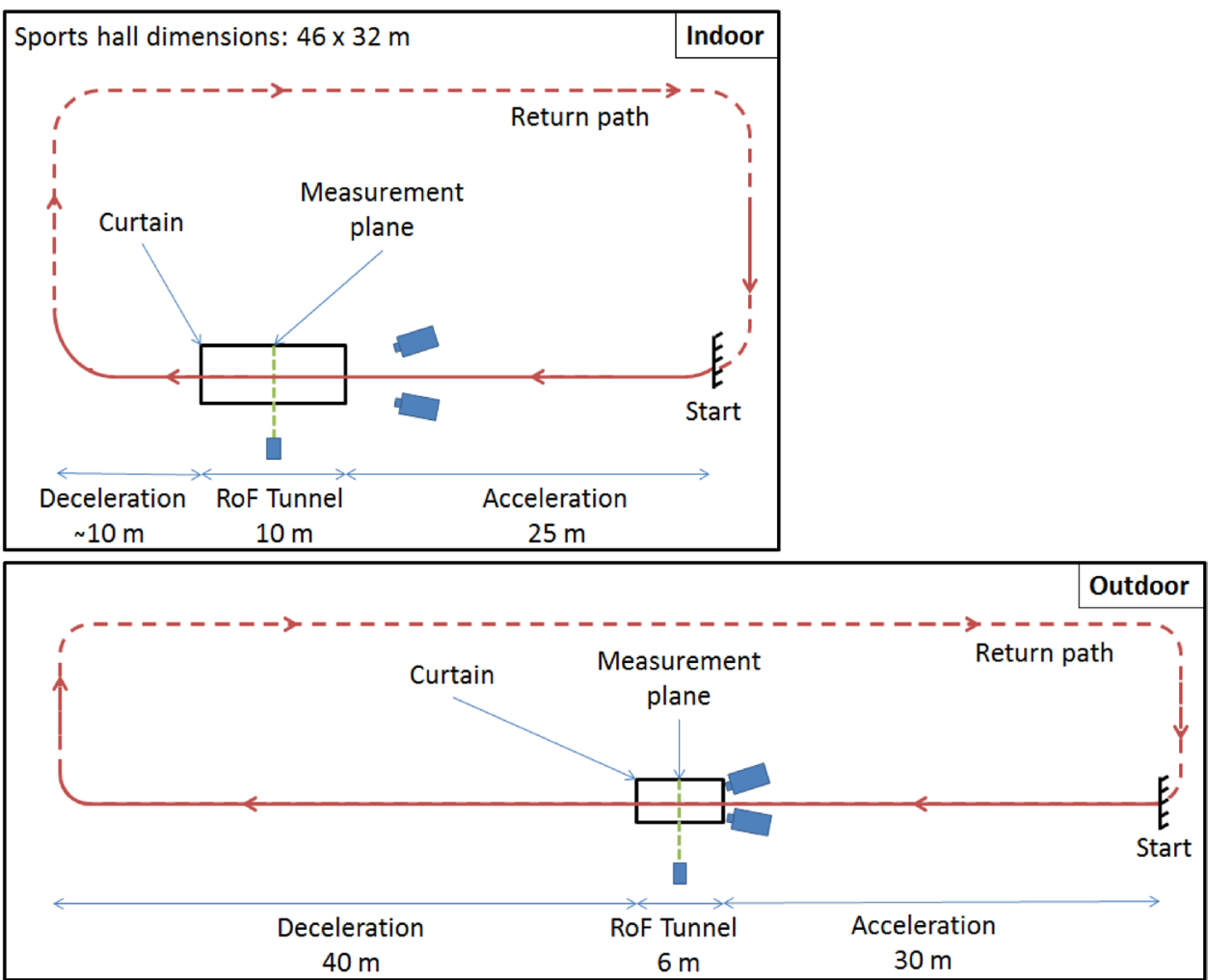


is assumed for the control volume approach. The floor is covered with a thin carpet (polypropylene, $3 \mathrm{~mm}$ ) to avoid ground slipperiness due to the PIV seeding.

A photograph of the setup during experiments is shown in Figs. 3 and 4. During the indoor experiment, the cameras were positioned $6 \mathrm{~m}$ upstream of the duct entrance.

Although the two experiments have similar acceleration length before the measurement plane (Fig. 2), the limited available braking length in the indoor experiments requires conducting the tests at lower velocity $(5.3 \mathrm{~m} / \mathrm{s})$. The crank angle $\varphi$ is defined as the angular position of the right foot (forward) with respect to horizontal crank position (Fig. 5-left).

In both the cases, measurements are conducted with the cyclist in upright and time-trial position (see Fig. 5). Following Crouch et al. (2014), the pedaling frequency (cadence) is normalized with the advancing speed, $k=\frac{2 r \pi f}{u_{\mathrm{c}}}$, where $r$ is the bike crank length, $f$ the cadence and $u_{\mathrm{C}}$ the cyclist velocity, as reported in Table 1 . The reduced frequency is $k=0.12$ indoor and $k=0.23$ outdoor, respectively.

\subsection{PIV instrumentation, imaging and data processing}

Velocity measurements are performed with a large-scale stereoscopic-PIV system. The experimental parameters are presented in Table 2. Neutrally buoyant helium-filled soap bubbles (HFSB) are used with an average diameter between 0.3 and $0.4 \mathrm{~mm}$, providing sufficient light scattering to visualize a field of view (FOV) of the order of $4 \mathrm{~m}^{2}$. The tracers
Fig. 3 Experimental setup in indoor conditions
Fig. 4 Experimental setup in outdoor conditions
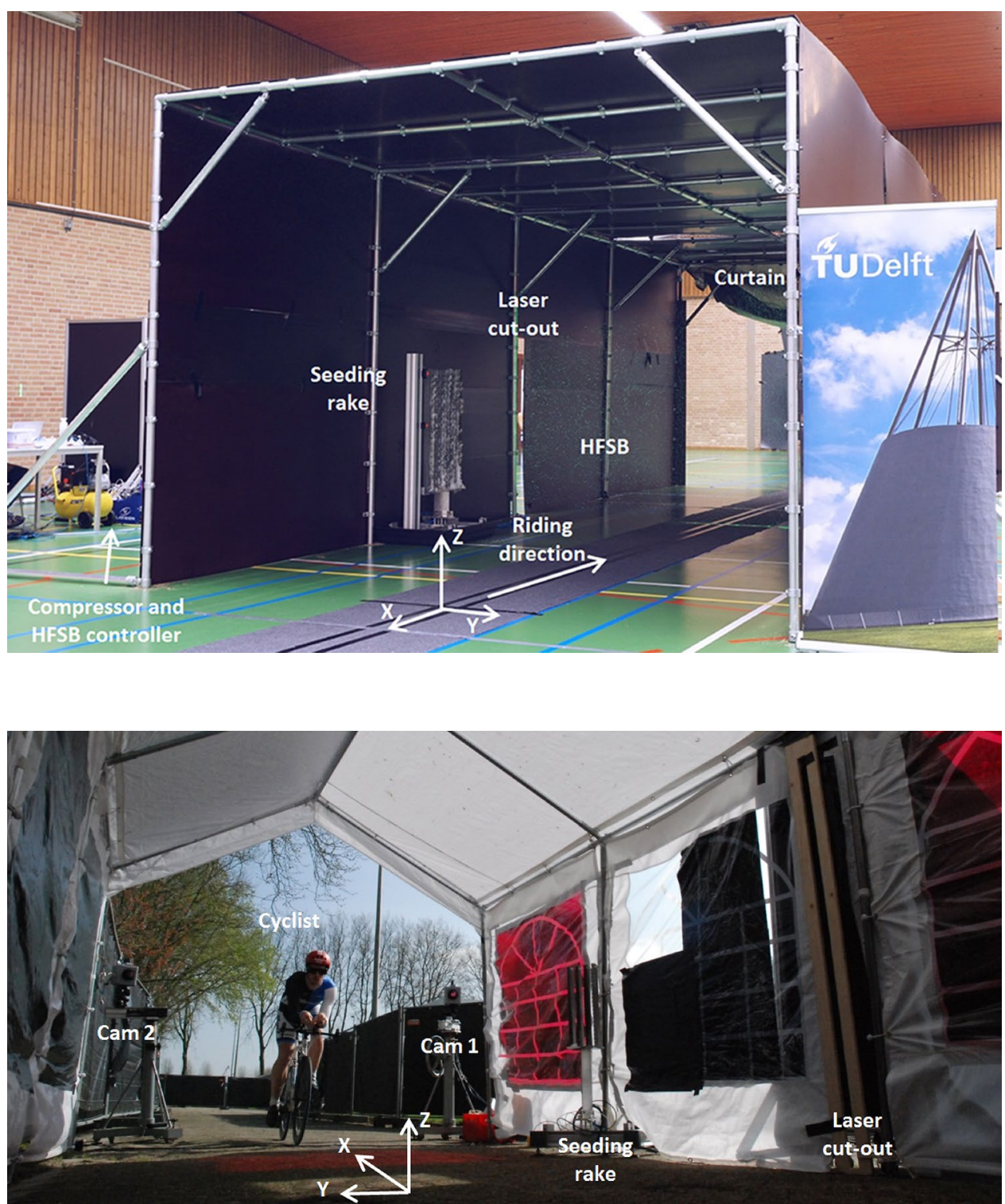

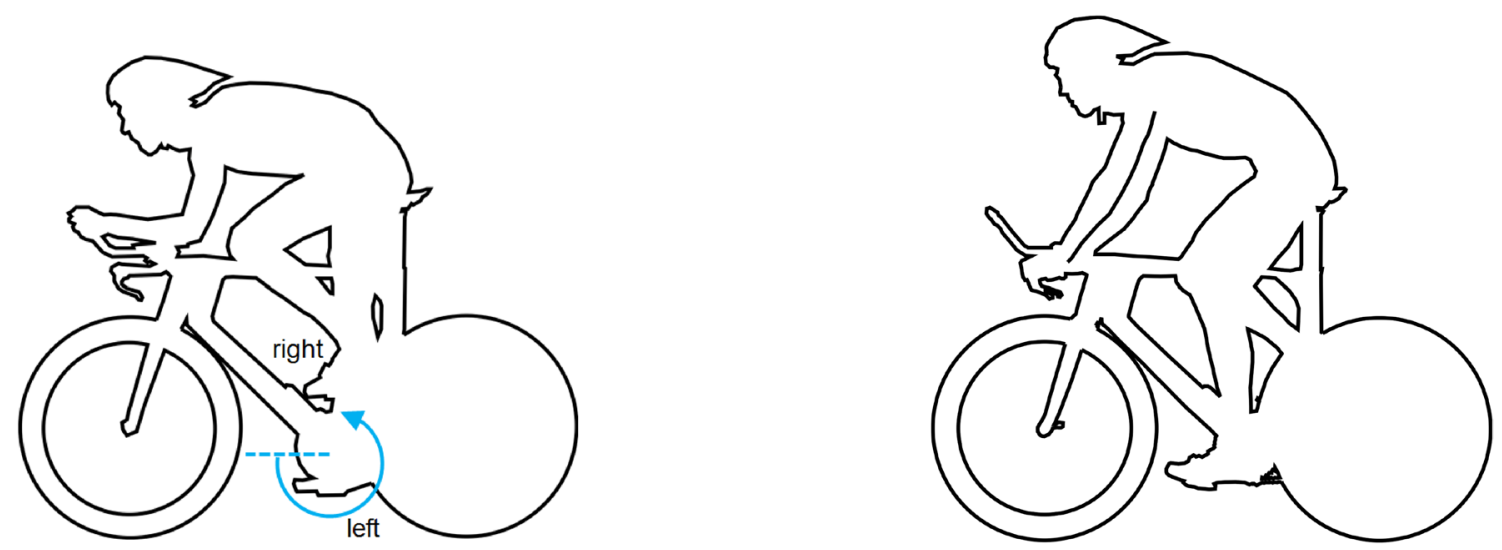

Fig. 5 Cyclist posture during the experiments: time trial (left) and upright (right)

Table 2 Equipment and setup parameters

\begin{tabular}{|c|c|c|c|c|c|}
\hline \multicolumn{6}{|l|}{ Equipment } \\
\hline Purpose & Instrument & \multicolumn{2}{|c|}{ Indoor (Low-speed PIV) } & \multicolumn{2}{|l|}{ Outdoor (High-Speed PIV) } \\
\hline \multirow[t]{3}{*}{ Imaging } & Cameras & \multicolumn{2}{|c|}{$\begin{array}{l}\text { LaVision Imager LX 2MP interline } \\
\text { CCD }(1628 \times 1236 \text { pixels, pixel pitch } \\
4.4 \mu \mathrm{m}, 14 \text { bits })\end{array}$} & \multicolumn{2}{|c|}{$\begin{array}{l}\text { Photron FastCAM SA1 cameras (CMOS, } \\
1024 \times 1024 \text { pixels, pixel pitch } 20 \mu \mathrm{m} \text {, } \\
12 \text { bits) }\end{array}$} \\
\hline & Objectives & \multicolumn{2}{|c|}{$2 \times$ AF Nikon $f=35 \mathrm{~mm}$} & \multicolumn{2}{|l|}{$2 \times$ Nikon $f=50 \mathrm{~mm}$} \\
\hline & Others & \multicolumn{2}{|c|}{ Bandpass filter (532 nm) } & \multicolumn{2}{|l|}{ Bandpass filter (532 nm) } \\
\hline Illumination & Laser & \multicolumn{2}{|c|}{$\begin{array}{l}\text { Quantel Evergreen } 200 \\
\text { Nd:YAG }(2 \times 200 \mathrm{~mJ} \text { at } 15 \mathrm{~Hz})\end{array}$} & \multicolumn{2}{|l|}{$\begin{array}{l}\text { Quantronix Darwin Duo Nd:YLF } \\
(2 \times 25 \mathrm{~mJ} \text { at } 1 \mathrm{kHz})\end{array}$} \\
\hline \multirow[t]{2}{*}{ Seeding } & Tracer particles & \multicolumn{2}{|c|}{ Helium-filled soap bubbles } & \multicolumn{2}{|l|}{ Helium-filled soap bubbles } \\
\hline & Seeding system & \multicolumn{2}{|c|}{200 nozzles } & \multicolumn{2}{|l|}{80 nozzles } \\
\hline \multicolumn{6}{|c|}{ Imaging and acquisition parameters } \\
\hline \multirow[t]{2}{*}{ Purpose } & \multicolumn{2}{|c|}{ Parameter } & \multicolumn{2}{|l|}{ Indoor } & Outdoor \\
\hline & \multicolumn{2}{|c|}{$X$ (thickness) $[\mathrm{cm}]$} & 4 & & 3 \\
\hline \multirow[t]{2}{*}{ Field of view } & \multicolumn{2}{|c|}{$Y$ (Width) $[\mathrm{m}]$} & 1.8 & & 1.8 \\
\hline & \multicolumn{2}{|c|}{$Z$ (Height) $[\mathrm{m}]$} & 2.4 & & 1.8 \\
\hline \multirow[t]{5}{*}{ Imaging } & \multicolumn{2}{|l|}{$f_{\#}$} & 5.6 & & 5.6 \\
\hline & \multicolumn{2}{|c|}{ Magnification } & 0.00297 & & 0.0111 \\
\hline & \multicolumn{2}{|c|}{ Object distance (m) } & 11.5 & & 4 \\
\hline & Digit & $\mathrm{n}(\mathrm{mm} / \mathrm{px})$ & 1.5 & & 1.8 \\
\hline & Stere & & 28 & & 31 \\
\hline Measurement rate & $f_{\text {acq }}($ & & 8 & & 2000 \\
\hline Pulse separation & $\Delta t(\mathrm{n}$ & & 3 & & 0.5 \\
\hline Seeding concentration & Parti & y (ppp) & 0.11 & & 0.09 \\
\hline
\end{tabular}

are introduced in the measurement region by a seeding rake (see Figs. 3, 4) consisting out of 200 and 80 nozzles in the indoor and the outdoor experiment, respectively. A LaVision HFSB fluid supply unit (FSU) controls soap, air and helium rates towards the seeding rake. The tracers concentration achieved during experiments is of 5 bubbles $/ \mathrm{cm}^{2}$ and $3 \mathrm{bub}-$ bles $/ \mathrm{cm}^{2}$ for indoor and outdoor, respectively. Based on the previous studies (Scarano et al. 2015; Faleiros et al. 2019), in the above conditions, a tracers response time in the order of $10-100 \mu$ s is expected. The tracers Stokes number based on cyclist velocity and torso length is in the order of $10^{-3}$.

In the indoor experiment, a low-repetition rate PIV system is used, whereas the outdoor experiment features high-speed PIV (see Table 2 for specifications). The results are not affected by the selection of the hardware, which is different only due to the availability at the time of the 
experiment. The low-speed system benefits from the higher pulse energy and sensor resolution with well-resolved particle images (diffraction disk covered with approximately 2 pixels). On the other hand, the high-speed system offers three orders of magnitude higher temporal resolution, enabling more advanced data processing, at the cost, however, of a lower imaging resolution (diffraction disk imaged over 0.5 pixels). The pulse separation with the low-speed system is chosen considering the out-of-plane loss-of-correlation factor (Keane and Adrian 1992). A cross-correlation analysis with multigrid image deformation (Scarano and Riethmuller 2000 ) is employed. A typical recording of particle images is shown in Fig. 6 for both experiments. The more controlled environment conditions in the indoor experiment result in a more uniform dispersion of the tracers and PIV images with homogeneous concentration. Achieving uniform seeding distribution in the outdoor experiment is hampered by the effect of wind gusts. From the raw PIV images, the cyclist's crank angle at the moment of the passage through the laser sheet is determined with an accuracy of $\pm 10^{\circ}$.

\subsection{Data processing}

The recorded images are analyzed with the LaVision DaVis 8 software. The pre-processing removes background light by subtracting the minimum intensity over time at each pixel. The recordings from the indoor experiment are analyzed with dual-frame cross-correlation. The time separation between frames is set to $3 \mathrm{~ms}$. A sliding sum-of-correlation algorithm (Sciacchitano et al. 2012) is employed for the outdoor experiment. For the latter, the analysis performs an average of the correlation maps from seven pairs of frames sliding a time interval of $3.5 \mathrm{~ms}$. The time separation between frames is set to $2 \mathrm{~ms}$. To quantify the range of resolvable velocity scales, the dynamic velocity range (DVR) is determined as the ratio between the maximum velocity in the near wake of the cyclist and the standard deviation of the velocity distribution in the quiescent flow prior to the cyclist's passage. Details of the image processing parameters and estimates of the measurement dynamic range are summarized in Table 3.

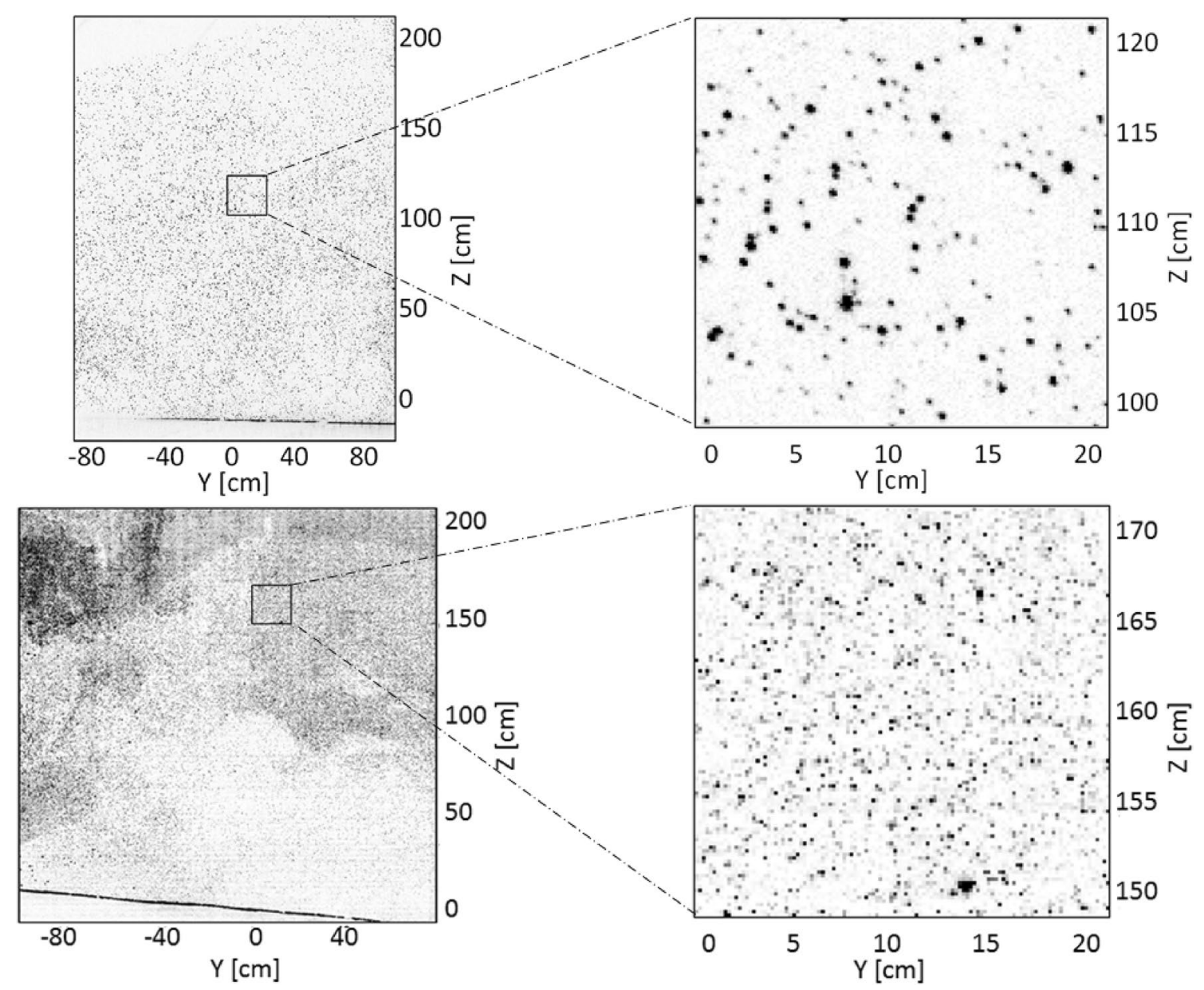

Fig. 6 Digital recordings of particle images (left) and detail of particle images (right). Indoor (top) and outdoor (bottom) experiment. Intensity gray-scale inverted for clarity 
The drag force evaluation after one passage of the cyclist is obtained via Eq. (2). The velocity field prior to the passage of the cyclist is significantly weaker than in his wake. Averaging the measurements before passage over a short time interval ( $1.25 \mathrm{~s}$ and $0.1 \mathrm{~s}$ for the indoor and outdoor experiment, respectively) reduces the effect of measurement noise in the determination of $u_{\mathrm{env}}$. To further reduce the measurement noise in the drag estimate, a wake contouring approach is applied which isolates the cyclist's wake from the outer flow region. The wake is defined as the flow region whose velocity is below a certain fixed percentage (5\% in the present case) of the minimum velocity in the flow field. Such region is then dilated by two adjacent vectors to include also the shear layers, thus obtaining the outlet surface $S_{2}$ of Eq. (2). The inlet surface $S_{1}$ is obtained by shrinking $S_{1}$ in all directions up to the point that the conservation of mass is satisfied.

The cyclist's speed is monitored measuring the bicycle transit time across the light sheet. In the indoor experiment, a magnetic sensor provides the cyclist speed in real time, additionally.

The wake past the cyclist exhibits unsteady behavior. Consequently, also the evaluation of the drag force yields temporal variations. A statistically significant estimate of the cyclist's average drag is produced by ensemble averaging (Eq. 3) the velocity field obtained from 10 and 28 repeated measurements for the outdoor and indoor conditions, respectively.

Two main repeatability issues are identified that require a specific treatment of the instantaneous data to retrieve ensemble average flow fields: (1) since the cyclist crosses the measurement plane at a different $Y$ coordinate for every passage, the measured velocity field is relocated in the $Y$-direction to compensate for such shift; (2) the relative distance between cyclist and measured wake planes is not exactly the same among different passages; the exact streamwise relocation is obtained examining the position of the cyclist when he crosses the measurement plane. For the latter problem, the high-speed PIV system resolves the motion of the cyclist within few millimeters in the streamwise direction; therefore, any error associated with variations of the relative distance between cyclist and wake planes can be neglected.
The results are presented in the coordinate system as shown in Figs. 3 and 4 with $t=0$ defined as when the rearmost point of the saddle crosses the laser sheet. To make the comparison between results from both experiments possible, the flow field variables and time are made dimensionless in the following way:

Dimensionless streamwise velocity $u_{x}^{*}: u_{x}^{*}=\frac{u_{\mathrm{wake}}-u_{\mathrm{env}}-\left|u_{\mathrm{C}}\right|}{\left|u_{\mathrm{C}}\right|}$,

Dimensionless streamwise vorticity $\omega_{x}^{*}: \omega_{x}^{*}=\frac{\omega_{x} \cdot c}{\left|u_{\mathrm{C}}\right|}$,

Dimensionless time $t^{*}: t^{*}=\frac{t \cdot\left|u_{\mathrm{C}}\right|}{c}$.

The dimensionless streamwise velocity $u_{x}^{*}$ is written in the frame of reference of the cyclist, meaning that when $u_{x}^{*}=0$, the velocity deficit is equal to the cyclist velocity, and when $u_{x}^{*}=1$ there is no velocity deficit (equivalent to freestream conditions).

The uncertainty of the estimated $C_{\mathrm{d}} A$ values is analyzed a posteriori, based on the standard deviation of the instantaneous drag area estimates, and the number of independent samples (considering both the number of passages of the cyclist, and the number of independent flow measurements in the wake of a cyclist during one passage). A detailed analysis of the measurement uncertainty and drag resolution of the Ring of Fire system for small-scale applications is reported in a recent work of Terra et al. (2018), where the effect of simplifications in the conservation of momentum equation is considered.

\subsection{Measurement procedure}

Before the passage of the athlete, the duct curtains are closed and the HFSB accumulate for approximately $2 \mathrm{~min}$. Atmospheric wind conditions require continuous operation of the seeding generator for the outdoor experiment. Instead, in the indoor experiment the bubbles production is paused prior to the passage of the cyclist and the momentum disturbance introduced by the seeding rake micro jets decays.
Table 3 Image processing parameters and expected dynamic range of measurements

\begin{tabular}{lll}
\hline Parameter & Indoor & Outdoor \\
\hline Correlation Algorithm & Dual-frame cross-correlation & Sliding sum-of-correlation \\
Interrogation windows & $64 \times 64 \mathrm{px}\left(95 \times 95 \mathrm{~mm}^{2}\right)$ & $64 \times 64 \mathrm{px}\left(116 \times 116 \mathrm{~mm}^{2}\right)$ \\
Overlap factor & $75 \%$ & $75 \%$ \\
Vector pitch & $24 \mathrm{~mm}$ & $29 \mathrm{~mm}$ \\
Dynamic spatial range & 25 & 15 \\
Dynamic velocity range & 250 & 100 \\
\hline
\end{tabular}


The cyclist starts from the same predefined distance and crank angle for each passage, to have well-matching athlete posture (leg position) in the measured area between passages. In the indoor experiment, the image acquisition is triggered by a photoelectric sensor, while the user manually triggers the image acquisition in the outdoor experiment. Transferring the acquired images to mass storage requires 5 min with the high-speed PIV system, whereas typically 40 image pairs are recorded with the low-speed PIV system permitting to repeat the experiment within $1 \mathrm{~min}$.

\section{Results}

\subsection{Quantitative flow visualization}

\subsubsection{Air flow conditions before cyclist transit}

The conditions before the passage of the cyclist rarely exhibit fully quiescent air. The environmental flow motions feature a velocity $u_{\text {env }}$, which is in general non-zero, nonuniform and non-stationary, mostly due to external conditions and the seeding injection. An instantaneous flow field before the cyclist's passage is illustrated in Fig. 7 for both the indoor (left) and outdoor (right) experiments. To reduce the noise in the data, the velocity is averaged in time during $1.25 \mathrm{~s}$ (indoor) and $0.1 \mathrm{~s}$ (outdoor) before the passage of the athlete.
The indoor experiment was performed in a closed, thus, quieter environment; whereas during the outdoor experiment, the presence of moderate wind $(0.5-1 \mathrm{~m} / \mathrm{s})$ could only be partly attenuated by the walls of the tunnel. This is clearly visible in Fig. 7, where the environment velocity is of the order of a $5 \mathrm{~cm} / \mathrm{s}$ in the indoor experiment and attains $30 \mathrm{~cm} / \mathrm{s}$ outdoor.

The velocity distribution prior to the passage is taken into account for the drag computation via Eq. (2) as it contributes to the overall momentum budget, as also discussed by Terra et al. (2018). Furthermore, unsteady effect may influence the interaction of the wake with the initial velocity field, resulting in variations of the measured drag. The latter effects, however, are neglected and cannot be directly observed with the current experimental apparatus.

\subsubsection{Velocity field in the cyclist wake}

The flow fields in the wake of the cyclist are discussed for the indoor upright and time-trial configuration as well as for the outdoor time-trial configuration. Figure 8 shows a comparison of the instantaneous streamwise velocity $u_{x}^{*}$ at $t^{*}=3$. Note that the cyclist contours in Fig. 8 are meant to indicate the general cross section of the athlete and do not reproduce the exact position of the legs. The development of both an indoor as well as an outdoor instantaneous wake over time is available online as supplementary material.

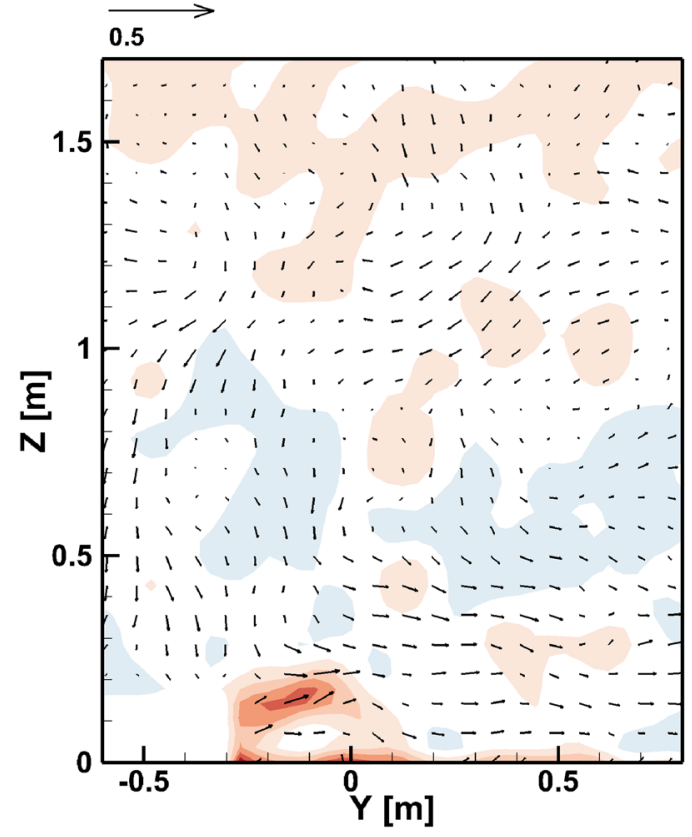

Fig. 7 In-plane velocity vector field from an instantaneous run, averaged over a short time sequence $(1.25 \mathrm{~s}$ and $0.1 \mathrm{~s}$ for the indoor and outdoor experiments, respectively) and color contours of streamwise

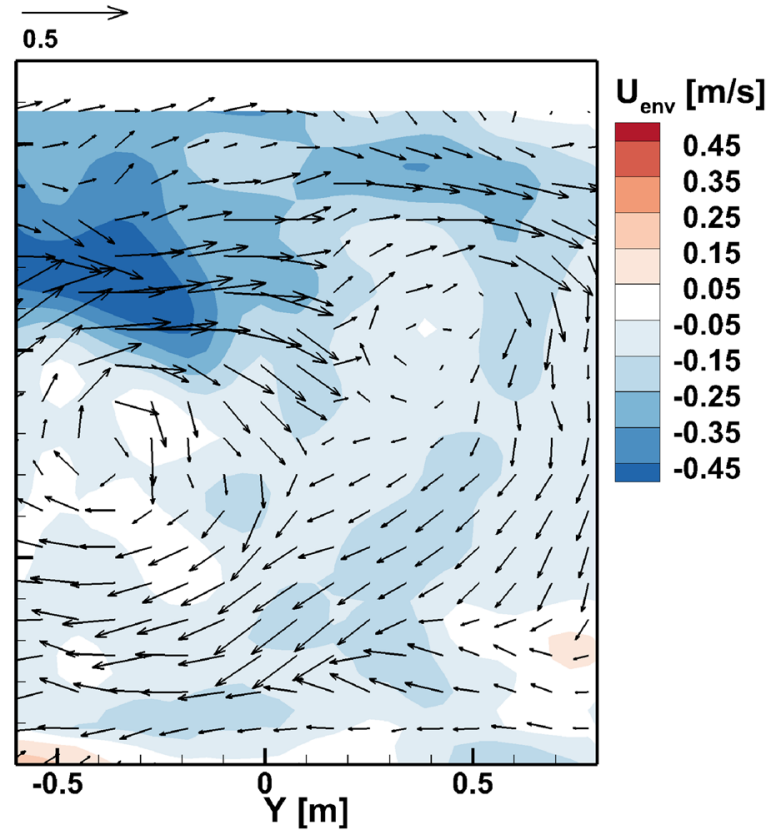

velocity component. Indoor conditions: $3.5 \mathrm{~m}(0.7 \mathrm{~s})$ in front of the cyclist (left); outdoor conditions: $2 \mathrm{~m}(0.25 \mathrm{~s})$ in front of the cyclist (right) 

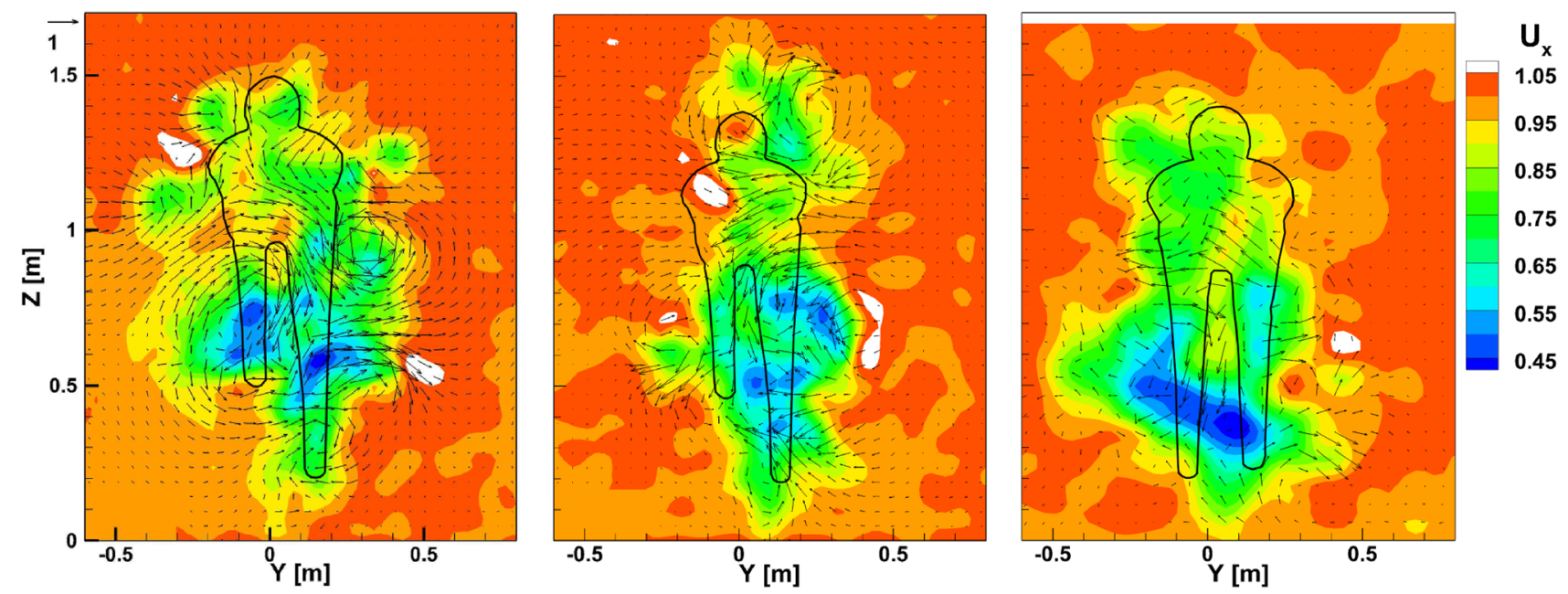

Fig. 8 Dimensionless instantaneous streamwise velocity $u_{x}^{*}$ at $t^{*}=3$. Left: indoor experiment upright position $\left(\varphi=65^{\circ}\right)$; middle: indoor experiment time-trial position $\left(\varphi=65^{\circ}\right)$; right: outdoor experiment

First, a comparison between time-trial (Fig. 8-left) and upright positions (Fig. 8-middle) for the indoor experiment is given. The magnitude and location of the peak momentum deficit are similar in both cases. The out-of-plane velocity contour of the wake $\left(u_{x}^{*}=0.95\right)$, however, is clearly wider for the upright case. Interestingly, it has the same height for the time-trial position as it has for the upright position, despite a higher height of the cyclist in upright position.

Next, the time-trial position is compared between the indoor (Fig. 8-middle) and outdoor experiments (Fig. 8right). The wake observed in the outdoor experiment is wider and shows a slightly higher peak momentum deficit. Despite that the heights of both cyclists in time-trial position were very similar, in the indoor experiment, the $u_{x}^{*}=0.95$ contour is consistently higher (see also Fig. 9). A reason for this can be the different inclination angle of the torso of both cyclists, generating a different amount of downwash over the back.

The temporal development of the ensemble average streamwise velocity field $\left(\overline{u_{x}^{*}}\right)$ past the cyclist is shown in Fig. 9. The ensemble average is obtained from 28 and 10 individual runs from, respectively, the indoor and outdoor experiments. The maximum deficit in the wake $(\sim 45 \%)$ is observed at the shortest time delay after the passage. The deficit is not uniformly distributed and attains its maximum behind the legs. The turbulent diffusion causes a rapid redevelopment of the flow in the wake, as it is seen for the individual runs as well. Considering its boundary by the contour where the streamwise velocity attains $95 \%$ of the undisturbed value, one observes that the flow entrainment smoothens the fine details of the streamwise velocity distribution and internally to the wake, the peak velocity deficit reduces. The diffusion process causes the wake to time-trial position $\left(\varphi=320^{\circ}\right)$. Instantaneous wake development over time available online

exceed the measurement region, with consequences on the uncertainty of the drag estimate. This occurs earlier for the outdoor experiment $\left(t^{*} \sim 9\right)$ than for the indoor experiment $\left(t^{*} \sim 13\right)$, which is ascribed to the higher intensity of velocity fluctuations in the surrounding environment. The higher acquisition frequency of the outdoor experiment provides a more detailed look into the temporal development of the wake, however, at the cost of a lower accuracy and higher amount of erroneous vectors.

Next to the out-of-plane velocity, the similarity between the flow fields is also assessed by looking at the in-plane streamlines. It is apparent that the primary features are consistent throughout Fig. 9, in that, close to the cyclist, a strong downwash exists near the vertical centreline. It can be reasoned that this characteristic is responsible for the downward movement over time of the wake structure. Furthermore, a strong inwash between $0.8 \mathrm{~m}$ and $1.2 \mathrm{~m}$ from the floor is induced by the main hip vortices in both experiments, which is further increased by the head vortices as seen in Fig. 10. Over time, the hip/thigh vortex structure seems to outlast the smaller vortex structures, which in turn means that the former will dominate the wake behavior in the far wake. There, the induced inwash causes a narrowing of the upper wake, while the broadening of the lower wake structure can be assigned to the induced outwash by the vortex pair, as well as the present ground, which constrains the downwash.

The analysis of the wake in terms of vorticity elucidates some of the characteristic aspects of the flow developing around and past the cyclist. Figure 10 illustrates and compares the distribution of streamwise vortices as measured indoor (upright and time trial) and outdoor (time trial). Positive vorticity relates to counter-clockwise rotating vortices, while negative vorticity to clockwise ones. 

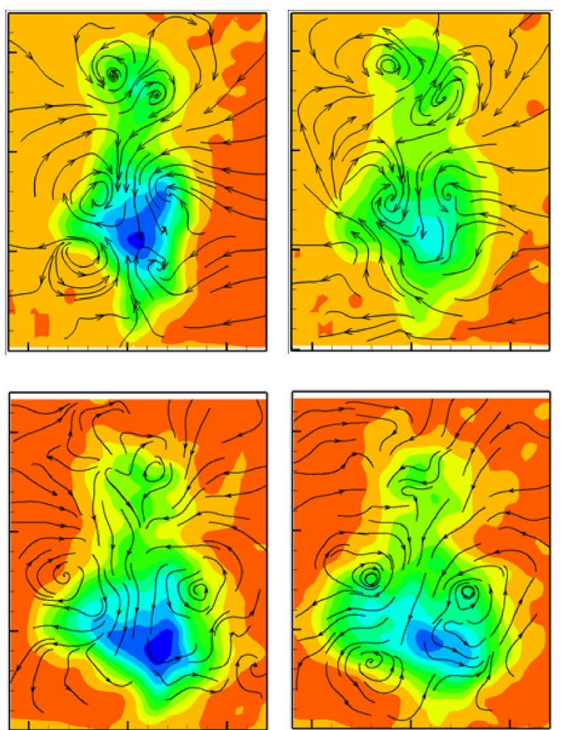

$\mathrm{t}^{*}=3$

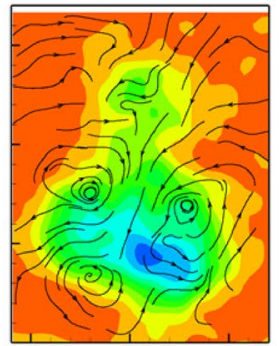

$\mathrm{t}^{*}=5$
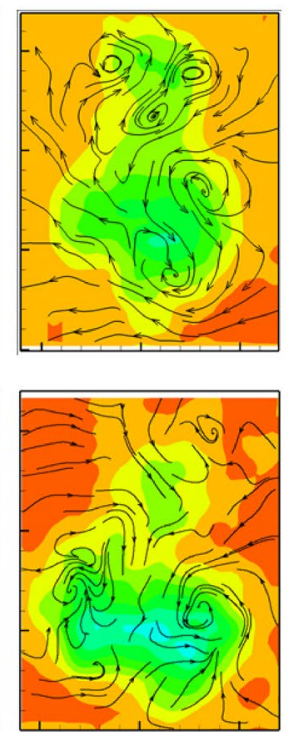

$\mathrm{t}^{*}=7$ $\bar{u}_{\mathrm{x}}^{\star}: \quad \begin{array}{llll}0.55 & 0.6 & 0.65 & 0.7\end{array}$
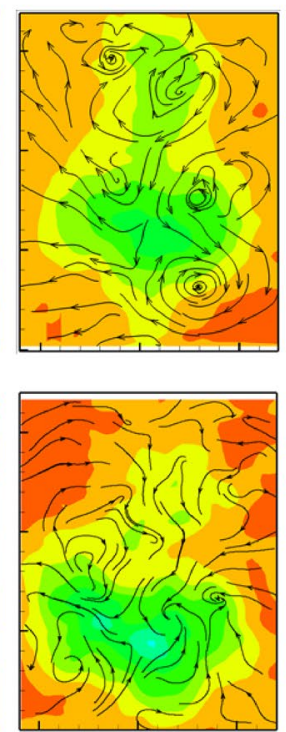

$\mathrm{t} *=9$ $\begin{array}{lllllll}0.75 & 0.8 & 0.85 & 0.9 & 0.95 & 1 & 1.05\end{array}$
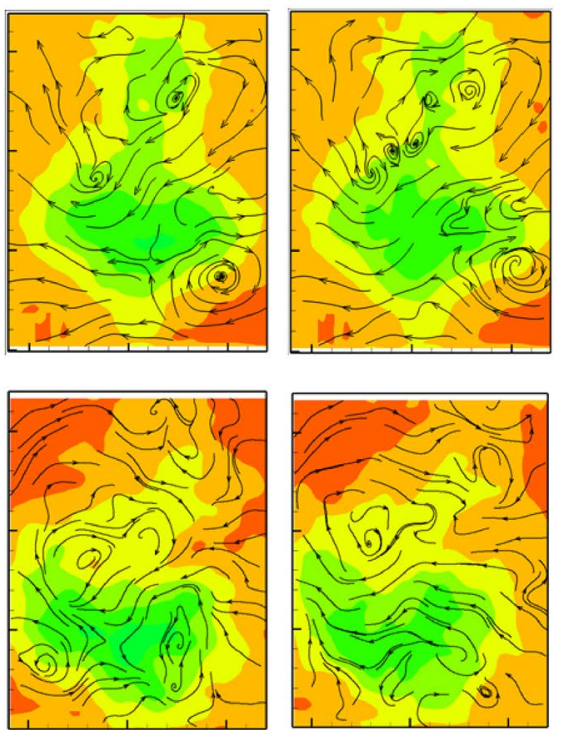

$\mathrm{t}^{*}=11$

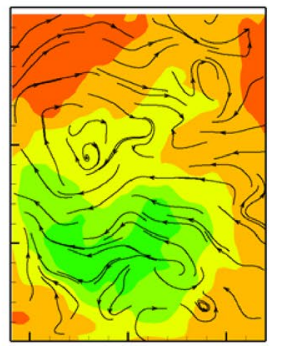

$\mathbf{t}^{*}=13$

Fig. 9 Development of the dimensionless ensemble average streamwise velocity over time. Indoor experiment (top) and outdoor experiment (bottom)
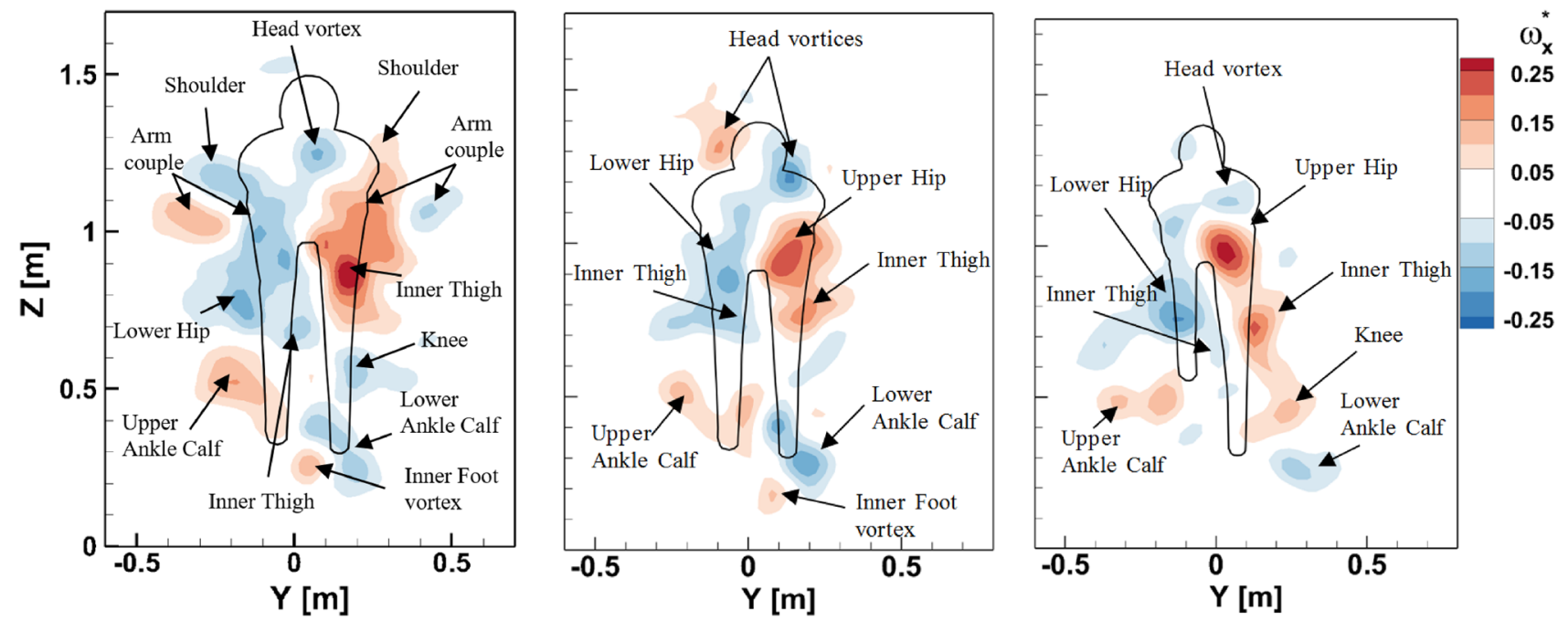

Fig. 10 Streamwise, ensemble averaged vorticity $\omega_{x}^{*}$ at $t^{*}=1.5$. Left: indoor experiment upright position $\left(\varphi=30^{\circ}\right)$; middle: indoor experiment time-trial position $\left(\varphi=30^{\circ}\right)$; right: outdoor experiment time-trial position $\left(\varphi=290^{\circ}\right)$

The flow structures characterizing the upright and timetrial wakes from the indoor experiment are compared in Fig. 10-left and -middle. There is substantial equivalence in the vortex structure strength and position, with the exception of the hip-thighs and the head vortices. In the former, the upright position shows higher vorticity on both sides. In the latter, the upright helmet vortices are negligible structures.
Moreover, the upright posture shows new large-scale structures, namely the shoulder vortex and the arm vortex couple. It is hypothesized that for each shoulder, one outer vortex is shed. Its generation mechanism is proper of what has been called as a 3D separation. In fact, on both sides, they are co-rotating with the hip vortices. This structure arises as a consequence of the very low pressure in the upper back 
of the cyclist. The arm vortex couples consist of a counterrotating vortex with respect to the shoulder vortex on the outside of the arm and a co-rotating one on the inside of the arm. They are assumed to be originated from the forward extended arms towards the brake hoods.

The vorticity field of the indoor and outdoor time-trial position exhibits an overall agreement, although some details are not exactly reproduced. This may be ascribed to the torso angle, not fully repeated during indoor and outdoor experiments. The vorticity structure presented in Fig. 10 also shows a good similarity with that reported in the studies of Crouch et al. (2014, 2016).

\subsection{Aerodynamic drag analysis}

\subsubsection{Ensemble average drag area}

Following the authoritative review article from Crouch et al. (2017), the drag results are presented as drag area $\left(C_{\mathrm{d}} A\right)$. In fact, the overall aerodynamic efficiency of the cyclist is governed by both frontal area of the cyclists and the bike and the drag coefficient (shape of the cyclist and bike). Based on Eq. (2) and on the procedures described in Sect. 3.3, the instantaneous drag area is computed for each passage as a function of the dimensionless time. In Fig. 11, the drag area evaluation is given for five passages with the cyclist in upright posture. In the outdoor experiment, half a crank cycle is spanned along $\Delta t^{*} \approx 4$, while along $\Delta t^{*} \approx 7.5$ for the indoor experiment.

For $t^{*} \leq 5$, the drag area computed via Eq. (2) is underestimated as the contribution of the static pressure in the measurement plane is wrongly estimated too close to the cyclist due to the larger velocity gradients (the in-plane gradients are modulated due to the limited spatial resolution; the outof-plane ones are completely neglected because stereo PIV is used). In case of the outdoor experiment, a $C_{\mathrm{d}} A$ plateau persists until approximately $t^{*} \leq 10$, when a sudden drop in the drag area occurs. This can be related to part of the wake moving out of the measurement domain in several runs. In the outdoor experiment, the external atmospheric conditions and a narrower field of view cause the problem. Moreover, the outdoor experiment generally exhibits larger fluctuations, especially in the near wake, which indicate a poorer control and repeatability of experimental conditions.

The comparison between two distinct postures of the athlete is shown in Fig. 12 to illustrate the overall sensitivity of the Ring of Fire system to macroscopic variations of the drag area. Together with the time-average $C_{\mathrm{d}} A$, a shaded band wide $2 \sigma_{C_{\mathrm{d}} A}$ represents the experimental uncertainty at $95 \%$ confidence level. Interestingly, although both the experiments were designed to obtain phase-locked average data, no clear cyclic trend depending on the crank angle is visible. This result differs from the findings of Crouch et al. (2014), who highlighted a $20 \%$ drag area variation with the crank angle, for a fixed $t^{*}$. This outcome shows that the wake diffusion and turbulent mixing is the main phenomenon affecting the streamwise wake trend.

\subsubsection{Time ensemble average drag area}

The measurements of drag area value for several configurations are summarized in Fig. 13. The interval $6 \leq t^{*} \leq 9$ is considered, where systematic errors due to the pressure term and wake exit from the measurement region can be neglected.

The drag area of the cyclist in the outdoor experiment is higher for both time-trial and upright positions. These results are in agreement with the wake contours in Figs. 8 and 9, where a wider contour with higher peak momentum deficit is observed for the outdoor cyclist. A relative difference between 20 and $35 \%$ is measured between time-trial and upright positions, which is in agreement with literature. The
Fig. 11 Instantaneous drag area measurements with the cyclist in upright posture
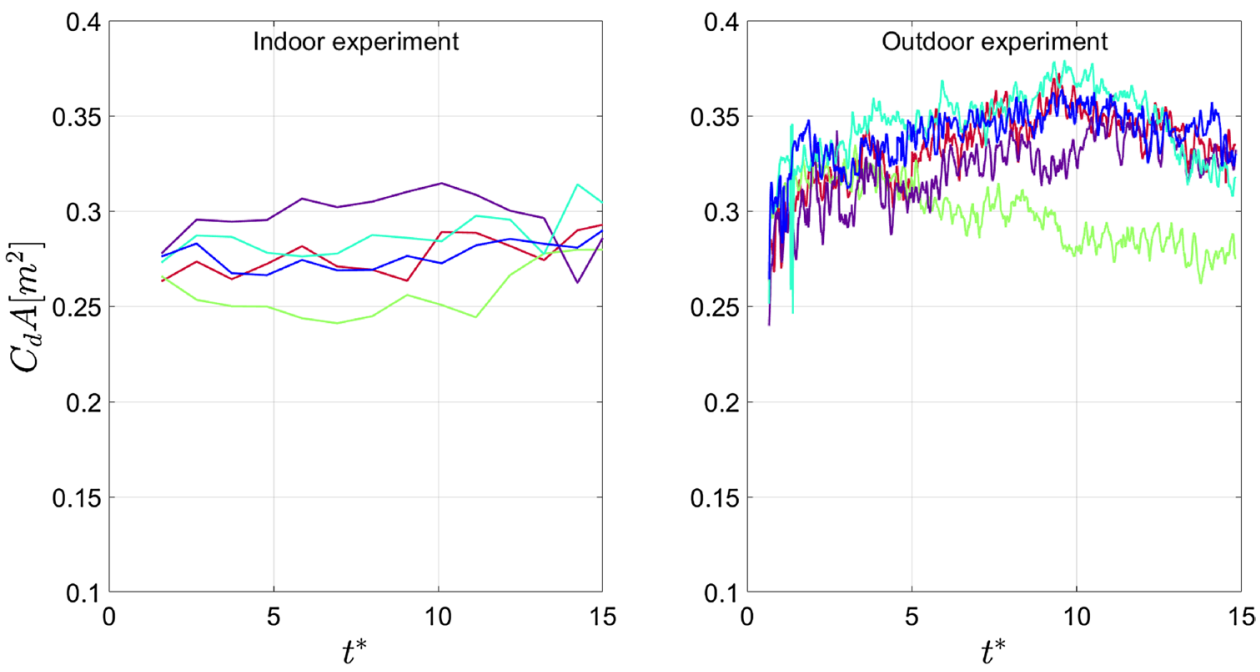
Fig. 12 Ensemble average drag area measurements for time-trial and upright postures
Fig. 13 Time ensemble average drag area; uncertainty bars for 95\% confidence interval. $N$ indicates the number of cyclist's passages per case
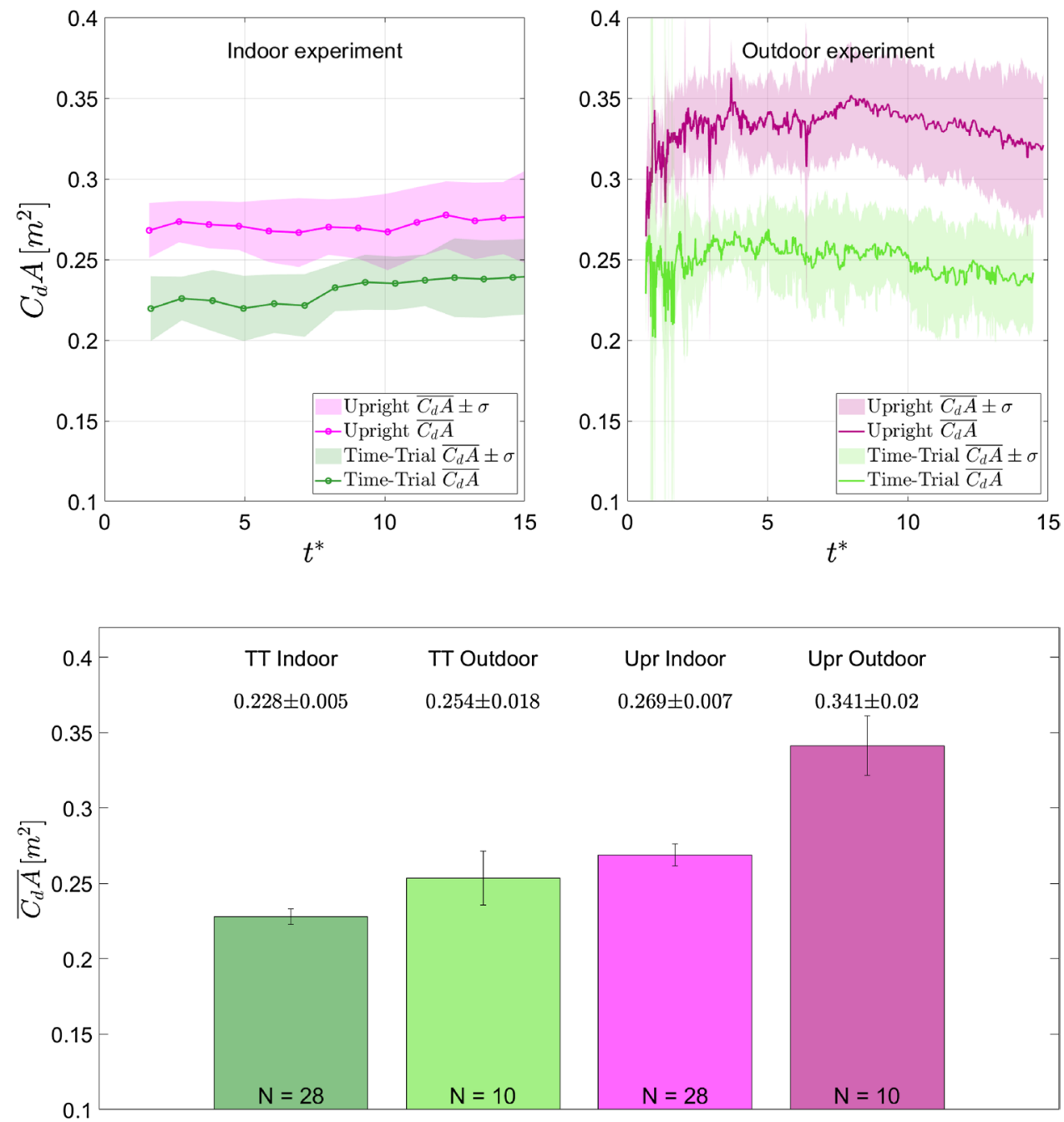

bigger difference between the two experiments is observed when comparing the mean drag area in upright position, with the outdoor experiment returning a higher $C_{\mathrm{d}} A$ value. It is hypothesized that this is due to a bigger difference in frontal area between the upright postures compared to the difference between the time-trial postures.

Finally, the current results are compared to the data collected from literature. The results of aerodynamic research in cycling exhibit a large scatter due to differences in riders, bicycle models, postures and garment and general experimental conditions. Figure 14 (top) and (bottom) compare drag areas versus velocity, measured in time-trial position and upright position, respectively, for different experiments and some computer simulations. Measurements obtained during races are obtained at velocity between 12 and $16 \mathrm{~m} / \mathrm{s}$. In our experiments, the limited space for accelerating and braking led to a lower velocity for the tests $(5-8 \mathrm{~m} / \mathrm{s})$. Conversely, Grappe (2009) showed that in the range of 5-20 m/s, the drag area of a cyclist remains approximately constant. The results from the current experiments fall within this large cloud of data and correlate favorably with wind tunnel and on-site experiments. In contrast, results from CFD simulations yield systematically lower values of drag area.

\section{Conclusions}

Large-scale stereo-PIV measurements are conducted to determine the aerodynamic drag of a moving cyclist in indoor and outdoor on-site conditions using the control volume approach. The flow is measured in the wake of a cyclist moving at $5 \mathrm{~m} / \mathrm{s}$ and $8 \mathrm{~m} / \mathrm{s}$ for, respectively the indoor and outdoor experiments. Instantaneous as well as ensemble average streamwise velocity fields have been obtained. Despite the differences between the two experiments in the cyclist geometry, bike model and the cycling speed, the flow fields in the near wake of the riders compare well between both experiments and literature. The instantaneous and ensemble average aerodynamic drag is evaluated via a control volume approach along the wake 
Fig. 14 Comparison of drag area of a cyclist in time-trial position (top) and in upright position (bottom) measured with different measurement techniques at different velocities. 1 Kyle (1991), 2 Hill (1993), 3 Broker and Kyle (1995), 4 Zdravkovic et al. (1996), 5 Martin et al. (1998), 6 Padilla et al. (2000), 7 Jeukendrup and Martin (2001), 8 Gibertini and Grassi (2008), 9 Garcia-Lopez et al. (2008), 10 Defraeye et al. (2010), 11 Underwood et al. (2011), 12 Chowdhury and Alam (2012), 13 Crouch et al. (2014), 14 Barry et al. (2015), 15 Crouch et al. (2016), 16 Blocken et al. (2016), 18 Griffith et al. (2012), 19 Blocken et al. (2013), 20 Defraeye et al. (2014), 21 Griffith et al. (2014), 23 Grappe et al. (1997), 24 Candau et al. (1999), 25 Peterman et al. (2015), 26 Nonweiler (1958), 27 Kyle and Burke (1984), 28 Fintelman et al. (2014), 29 Capelli et al. (1993), 30 de Groot et al. (1995), 31 Lim et al. (2011)
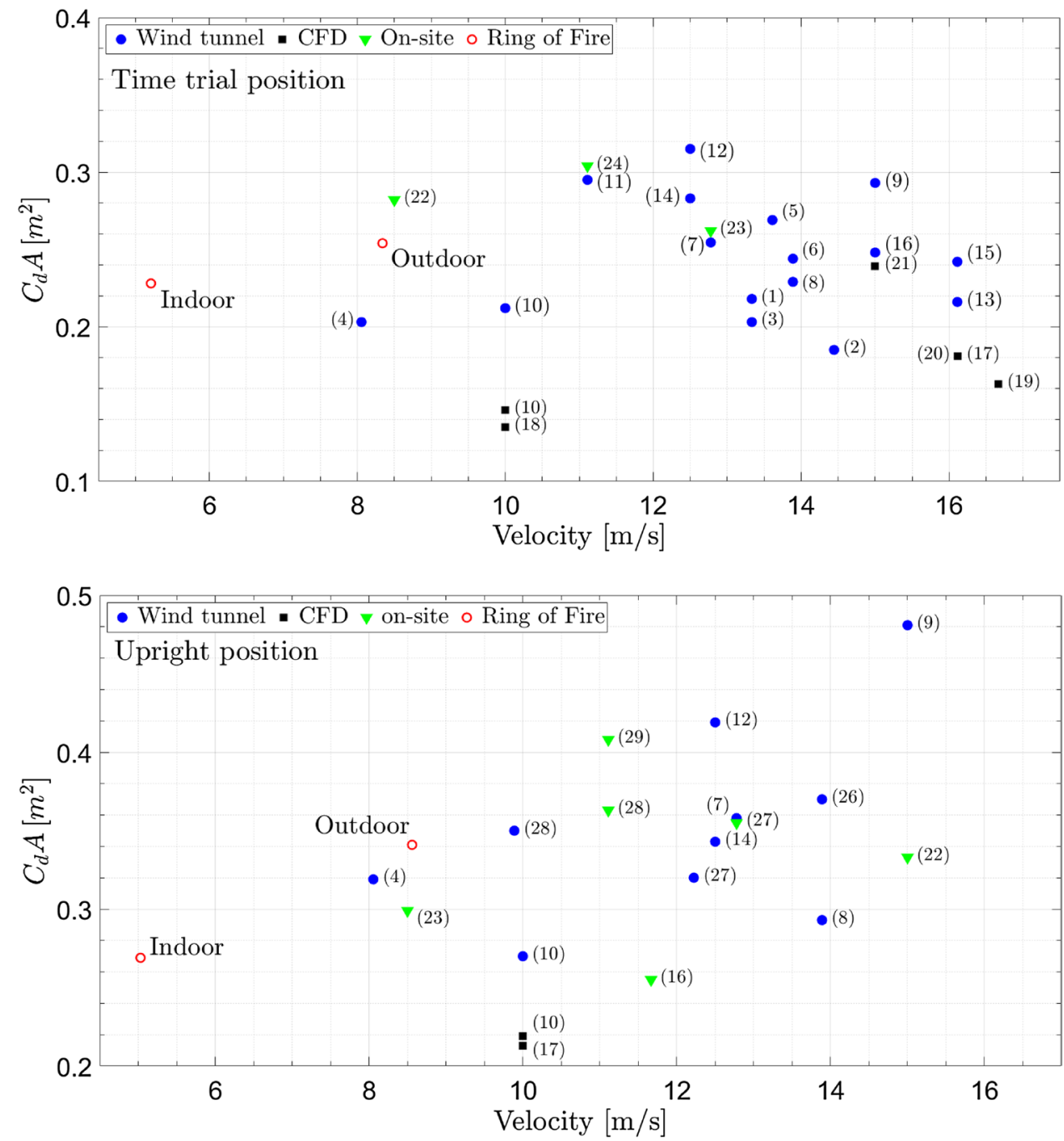

behind the cyclist, taking into account the non-uniform flow conditions prior to the cyclist's passage. A clear distinction in upright vs. time-trial ensemble average drag area is found for both experiments, with the upright posture yielding higher drag area by about $20-35 \%$ with respect to the time-trial posture.

Acknowledgements This research is supported by the Netherlands Organisation for Scientific Research (NWO) Domain Applied and Engineering Sciences (TTW), project 15583 "Enabling on-site sport aerodynamics with the Ring of Fire". The support of Team Sunweb who provided part of the cycling equipment for both experiments is kindly acknowledged.

Data and supplementary movies The data presented in this publication are available at: https://doi.org/10.4121/uuid:9ab877f9-1616-4f47$9 c 9 a-68 c 890 a 36 d 23$. Supplementary movies are available in the online version.

Open Access This article is distributed under the terms of the Creative Commons Attribution 4.0 International License (http://creativeco mmons.org/licenses/by/4.0/), which permits unrestricted use, distribution, and reproduction in any medium, provided you give appropriate credit to the original author(s) and the source, provide a link to the Creative Commons license, and indicate if changes were made.

\section{References}

Arnold VI (1989) Mathematical methods of classical mechanics, 2nd edn. Springer, Berlin (ISBN 0-387-96890-3)

Barlow JB, Rae WH, Pope A (1999) Low-speed wind tunnel testing. Wiley, Hoboken

Barry N, Burton D, Sheridan J, Thompson MC, Brown NAT (2015) Aerodynamic drag interactions between cyclists in a team pursuit. Sports Eng 18(2):93-103

Ben-Gida H, Kirchhefer A, Zachary T et al (2013) Estimation of unsteady aerodynamics in the wake of a freely flying European starling (Sturnus vulgaris). PLoS One. https://doi.org/10.1371/ journal.pone.0080086

Blocken B, Defraeye T, Koninckx E, Carmeliet J, Hespel P (2013) CFD simulations of the aerodynamic drag of two drafting cyclists. Comput Fluids 71:435-445 
Blocken B, Toparlar Y, Andrianne T (2016) Aerodynamic benefit for a cyclist by a following motorcycle. J Wind Eng Ind Aerodyn 155:1-10

Bosbach J, Kühn M, Wagner C (2009) Large scale particle image velocimetry with helium filled soap bubbles. Exp Fluids 46:539-547

Broker JP, Kyle CR (1995) Pursuit aerodynamics, Project 96: wind tunnel test results. USOC Sport Science and Technology Report, Colorado Springs, pp 1-46

Candau R, Grappe F, Ménard M, Barbier B, Millet GY, Hoffman M, Belli A, Rouillon JD (1999) Simplified deceleration method for assessment of resistive forces in cycling. Med Sci Sports Exerc 31(10): 1441-1447

Capelli C, Rosa G, Butti F et al (1993) Energy cost and efficiency of riding aerodynamic bicycles. Eur J Appl Physiol 67:144. https:// doi.org/10.1007/BF00376658

Chowdhury H, Alam F (2012) Bicycle aerodynamics: an experimental evaluation methodology. Sports Eng 15:73. https://doi. org/10.1007/s12283-012-0090-y

Coutanceau M, Bouard R (1977) Experimental determination of the main features of the viscous flow in the wake of a circular cylinder in uniform translation. Part 1. Steady flow. J Fluid Mech 79:231-256. https://doi.org/10.1017/S0022112077000135

Crouch T, Burton D, Brown N, Thompson M, Sheridan J (2014) Flow topology in the wake of a cyclist and its effect on aerodynamic drag. J Fluid Mech 748:5-35

Crouch T, Burton D, Brown N, Sheridan J, Thompson M (2016) Dynamic leg-motion and its effect on the aerodynamic performance of cyclists. J Fluids Struct 65:121-137

Crouch T, Burton D, LaBry ZA, Blair KB (2017) Riding against the wind: a review of competition cycling aerodynamics. Sports Eng 20:81-110

David L, Jardin T, Farcy A (2009) On the non-intrusive evaluation of fluid forces with the momentum equation approach. Meas Sci Technol 20:095401

De Groot G, Sargeant A, Geysel J (1995) Air friction and rolling resistance during cycling. Med Sci Sports Exerc 27(7):1090-1095

Defraeye T, Blocken B, Koninckx E, Hespel P, Carmeliet J (2010) Aerodynamic study of different cyclist positions: CFD analysis and full-scale wind-tunnel tests. J Biomech 43(7):1262-1268. https://doi.org/10.1016/j.jbiomech.2010.01.025

Defraeye T, Blocken B, Koninckx E, Hespel P, Verboven P, Nicolai B, Carmeliet J (2014) Cyclist drag in team pursuit: influence of cyclist sequence, stature, and arm spacing. J Biomech Eng 136(1):011005

Faleiros DE, Tuinstra M, Sciacchitano A, Scarano F (2019) Generation and control of helium-filled soap bubbles for PIV. Exp Fluids 60(3):40

Fintelman DM, Sterling M, Hemida H, Li FX (2014) The Effect of crosswinds on cyclists: an experimental study. Procedia Eng 72:720-725. https://doi.org/10.1016/j.proeng.2014.06.122

Garcia-Lopez J, Rodriguez-Marroyo JA, Juneau CE, Peleteiro J, Martinez AC, Villa JG (2008) Reference values and improvement of aerodynamic drag in professional cyclists. J Sports Sci 26(3):277-286

Gibertini G, Grassi D (2008) Cycling aerodynamics. In: Nørstrud $\mathrm{H}$ (ed) Sport aerodynamics. CISM International Centre for Mechanical Sciences, vol 506. Springer, Vienna. https://doi. org/10.1007/978-3-211-89297-8_3

Grappe F (2009) Resistance totale qui s'oppose au deplacement en cyclisme [Total resistive forces opposed to the motion in cycling]. In: Cyclisme et Optimisation de la Performance (2nd edn.) [Cycling and optimisation of performance]. De Boeck Université, Collection Science et Pratique du Sport, Paris, p. 604
Grappe F, Candau R, Belli A, Rouillon JD (1997) Aerodynamic drag in field cycling with special reference to the Obree's position. Ergonomics 40(12):1299-1311

Griffith MD, Crouch T, Thompson MC, Burton DM and Sheridan J (2012) Elite cycling aerodynamics: wind tunnel experiments and CFD. In: Brandner PA, Pearce BW (eds) Proceedings of the 18th Australasian fluid mechanics conference. Australasian Fluid Mechanics Society, Parkville, pp 1-4

Griffith M, Crouch T, Thompson M, Burton D, Sheridan J, Brown N (2014) Computational fluid dynamics study of the effect of leg position on cyclist aerodynamic drag. J Fluids Eng 136:101105

Hedenström A, Johansson LC (2015) Bat flight: aerodynamics, kinematics and flight morphology. J Exp Biol 218:653-663

Hill RD (1993) The design and development of the Lotus Sport Pursuit Bicycle. Proc Inst Mech Eng Part D J Automob Eng 207(4):285294. https://doi.org/10.1243/PIME_PROC_1993_207_194_02

Jeukendrup AE, Martin J (2001) Improving cycling performance-how should we spend our time and money. Sports Med 31(7):559-569

Jones BM (1936) Measurement of profile drag by the pitot-traverse method. Aeronautical Research Council R\&M Rept. 1688

Jönsson M, Loose S (2016) Experimental investigation of the flow field underneath a generic high-speed train and the effects of ground and train roughness. In: Dillmann A, Orellano A (eds) The aerodynamics of heavy vehicles III. ECI 2010. Lecture Notes in Applied and Computational Mechanics, vol 79. Springer, Cham. https://doi.org/10.1007/978-3-319-20122-1_2

Jux C, Sciacchitano A, Schneiders JFG, Scarano F (2018) Robotic volumetric PIV of a full-scale cyclist. Exp Fluids 59:74

Keane RD, Adrian RJ (1992) Theory of cross-correlation analysis of PIV images. Appl Sci Res 49:191

Kurtulus DF, Scarano F, David L (2007) Unsteady aerodynamic forces estimation on a square cylinder by TR-PIV. Exp Fluids 42:185196. https://doi.org/10.1007/s00348-006-0228-4

Kyle CR (1991) The effects of crosswinds upon time trials. Cycl Sci 3(3-4):51-56

Kyle CR, Burke ER (1984) Improving the racing bicycle. Mech Eng 106:34-35

Lim AC, Homestead EP, Edwards AG, Carver TC, Kram R, Byrnes WC (2011) Measuring changes in aerodynamic/rolling resistances by cycle-mounted power meters. Med Sci Sports Exerc 43(5):853-860

Lukes RA, Chin SB, Haake S (2005) The understanding and development of cycling aerodynamics. Sports Eng 8:59-74

Martin JC, Milliken DL, Cobb JE, McFadden KL, Coggan AR (1998) Validation of a mathematical model for road cycling power. J Appl Biomech 14:276-291

Nonweiler T (1958) The work production of man; studies on racing cyclists. J Physiol 141(1):8-9

Padilla S, Mujika I, Angulo F, Goiriena JJ (2000) Scientific approach to the 1-h cycling world record: a case study. J Appl Physiol 89:1522-1527

Peterman JE, Lim AC, Ignatz RI, Edwards AG, Byrnes WC (2015) Field-measured drag area is a key correlate of level cycling time trial performance. PeerJ 3:e1144. https://doi.org/10.7717/peerj .1144

Petrushov VA (1998) Improvement in vehicle aerodynamic drag and rolling resistance determination from coast-down tests. Proc Inst Mech Eng Part D J Automob Eng 212(5):369-380

Rival DE, Van Oudheusden B (2017) Load-estimation techniques for unsteady incompressible flows. Exp Fluids 58:20. https://doi. org/10.1007/s00348-017-2304-3

Scarano F, Riethmuller M (2000) Advances in iterative multigrid PIV image processing. Exp Fluids 29(Suppl 1):S051

Scarano F, van Wijk C, Veldhuis LLM (2002) Traversing field of view and AR-PIV for mid-field wake vortex investigation in a towing 
tank. Exp Fluids 33:950-961. https://doi.org/10.1007/s0034 8-002-0516-6

Scarano F, Ghaemi S, Caridi GCA et al (2015) On the use of heliumfilled soap bubbles for large-scale tomographic PIV in wind tunnel experiments. Exp Fluids 56:42

Sciacchitano A, Scarano F, Wieneke B (2012) Multi-frame pyramid correlation for time resolved PIV. Exp Fluids 53:1087

Terra W, Sciacchitano A, Scarano F (2017) Aerodynamic drag of a transiting sphere by large-scale tomographic-PIV. Exp Fluids 58:83. https://doi.org/10.1007/s00348-017-2331-0

Terra W, Sciacchitano A, Scarano F, van Oudheusden BW (2018) Drag resolution of a PIV wake rake for transiting models. Exp Fluids 59:120

Underwood L, Schumacher J, Burette-Pommay J, Jermy M (2011) Aerodynamic drag and biomechanical power of a track cyclist as a function of shoulder and torso angles. Sports Eng 14(2-4):147-154 van Oudheusden BW, Scarano F, Casimir EWF (2006) Non-intrusive load characterization of an airfoil using PIV. Exp Fluids 40:988. https://doi.org/10.1007/s00348-006-0149-2

Van Oudheusden BW, Scarano F, Roosenboom EWM, Casimiri EWF, Souverein LJ (2007) Evaluation of integral forces and pressure fields from planar velocimetry data for incompressible and compressible flows. Exp Fluids 43:153-162. https://doi.org/10.1007/ s00348-007-0261-y

Von Carmer CF, Heider A, Schröder A, Konrath R, Agocs J, Gilliot A, Monnier JC (2008) Evaluation of large-scale wing vortex wakes from multi-camera PIV measurements in free-flight laboratory. Part Image Velocim Top Appl Phys 112:377-394

Wilson DG (2004) Bicycling Science, 3rd edn. MIT Press, Cambridge

Zdravkovic MM, Ashcroft MW, Chisholm SJ, Hicks N (1996) Effect of cyclist's posture and vicinity of another cyclist on aerodynamic drag. In: Haake SJ (ed) The engineering of sport. Balkema, Rotterdam, pp 21-28

\section{Affiliations}

\section{Alexander Spoelstra $^{1}\left(\mathbb{D} \cdot\right.$ Luigi de Martino Norante $^{1} \cdot$ Wouter Terra $^{1} \cdot$ Andrea Sciacchitano $^{1} \cdot$ Fulvio Scarano $^{1}$}

Alexander Spoelstra

A.M.C.M.G.Spoelstra@tudelft.nl
1 Aerospace Engineering Department, Delft University of Technology, Kluyverweg 2, Delft 2629 HT, The Netherlands 